\title{
Effect of non-secretor/Lewis Phenotype on rotavirus-specific IgA response, vaccine take and clinical protection following monovalent rotavirus vaccination in Malawian infants
}

\section{Background}

Rotavirus (RV) vaccine efficacy is lower in Malawi and other high-burden, low-income settings than in lower-burden, higherincome settings. Several explanations for this have been proposed, but supportive data are limited.

Histo-blood-group-antigen(HBGA) phenotypes are associated with genotype-specific susceptibility to rotavirus infection. Evidence suggests non-secretor/Lewis negative infants may be resistant to G1P[8] infection. Since current vaccines are based on $\mathrm{P}[8]$ strains, this could limit vaccine efficacy.

We tested the hypothesis that non-secretor/Lewis negative phenotype leads to reduced vaccine virus replication, $\lg A$ response and clinical protection following monovalent G1P[8] rotavirus (RV1) vaccination in Malawian infants.

\section{Methods}

Population

Vaccine virus replication and immunogenicity were determined by a cohort study of infants receiving 2 doses of

RV1 at 6 and 10 weeks of age. Clinical protection was

determined by a cross-sectional case:control study comparing vaccinated infants $<12$ months with severe rotavirus gastroenteritis (RVGE) to age-matched, vaccinated, asymptomatic community controls.

Laboratory methods

Stool was collected on alternate days from days 4-10 post vaccine. Vaccine virus faecal shedding was determined by NSP2 RT-PCR with confirmatory VP6 qRT-PCR. Shedding was defined as detectable $R V 1$ from $\geq 2$ post vaccine samples.

Post-vaccine plasma rotavirus(RV)-specific lgA was determined by ELISA. Seroconversion defined as RV-specific $\lg A>20 \mathrm{U} / \mathrm{L}$ with $\geq$ fourfold rise in concentration among infants seropositive at baseline.

HBGA phenotype was determined by salivary ELISA. RVGE cases were confirmed by VP6 qRT-PCR. Genotype was determined by RT-PCR

Statistical methods

Log-binomial regression, logistic regression and conditional logistic regression were used to compare outcomes by HBGA phenotype in the cohort, genotype analysis and matched case:control studies respectively.
Results: Cohort Study

293 infants were recruited to the cohort study between May 2015 and August 2016, of whom 202 completed follow-up and were included in the analysis.

HBGA phenotype and vaccine virus faecal shedding The likelihood of vaccine virus faecal shedding (vaccine take) did not differ between infants with secretor compared to non-secretor phenotype, or between infants with Lewis positive compared to Lewis negative phenotype (Table 1).

Table 1

\begin{tabular}{|c|c|c|}
\hline $\begin{array}{l}\text { HBGA } \\
\text { phenotype }\end{array}$ & $\begin{array}{l}\text { Proportion of infants } \\
\text { with vaccine virus } \\
\text { faecal shedding } \\
\text { n, } \%(95 \% \mathrm{Cl})\end{array}$ & $\begin{array}{l}\text { Likelihood of vaccine } \\
\text { virus faecal shedding } \\
\text { RR }(95 \% \mathrm{Cl}) \text {, p value }\end{array}$ \\
\hline $\begin{array}{l}\text { Secretor } \\
\text { vs } \\
\text { Non-secretor }\end{array}$ & $\begin{array}{l}86 / 157,55(47-62 \%) \text { vs } \\
18 / 45,40(26-55 \%)\end{array}$ & $\begin{array}{l}1.4(0.9-2.0) \text { vs } \\
0.73(0.5-1.1) p=0.11\end{array}$ \\
\hline $\begin{array}{l}\text { Lewis positive } \\
\text { vs } \\
\text { Lewis negative }\end{array}$ & $\begin{array}{l}84 / 159,53(45-61 \%) \text { vs } \\
20 / 43,47(32-62 \%)\end{array}$ & $\begin{array}{l}1.1(0.8-1.6) \text { vs } \\
0.88(0.6-1.3) p=0.48\end{array}$ \\
\hline
\end{tabular}

HBGA phenotype and RV-specific IgA response

In 196 cohort infants with paired serology, the likelihood of

seroconversion did not differ between infants with secretor compared

to non-secretor phenotype, or between infants with Lewis positive

compared to Lewis negative phenotype (Table 2).

Table 2

\begin{tabular}{|c|c|c|}
\hline $\begin{array}{l}\text { HBGA } \\
\text { phenotype }\end{array}$ & $\begin{array}{l}\text { Proportion of infants } \\
\text { with } \\
\text { RV-specific IgA } \\
\text { seroconversion } \\
n, \%(95 \% C l)\end{array}$ & $\begin{array}{l}\text { Likelihood of RV- } \\
\text { specific IgA } \\
\text { seroconversion } \\
\text { RR }(95 \% \mathrm{Cl}) \text {, p valueb }\end{array}$ \\
\hline $\begin{array}{l}\text { Secretor } \\
\text { vs } \\
\text { Non-secretor } \\
\end{array}$ & $\begin{array}{l}27 / 151,27(21-35 \%) \\
6 / 45,13(6-27 \%)\end{array}$ & $\begin{array}{l}2.0(0.9-4.5) \\
0.5(0.2-1.1) p=0.08\end{array}$ \\
\hline $\begin{array}{l}\text { Lewis positive } \\
\text { vs } \\
\text { Lewis negative }\end{array}$ & $\begin{array}{l}35 / 149,24(17-31 \%) \\
12 / 47,26(15-40 \%)\end{array}$ & $\begin{array}{l}0.9(0.5-1.6) \\
1.1(0.6-1.9) p=0.77\end{array}$ \\
\hline
\end{tabular}

Results: Case-Control Study

5990 GE cases were screened and 319 RVGE cases identified between January 2015 February 2017. 123 eligible RVGE cases $<12$ months old were recruited, 119 with HBGA phenotype data. HBGA phenotype and genotype-specific susceptibility to RVGE Genotype data was available for 114 rotavirus cases, 28 asymptomatic rotavirus infections, and 43 rotavirus-negative community controls. Secretor phenotype was associated with increased risk of $\mathrm{P}[8]$ and $\mathrm{P}[4$ RVGE. Lewis negative phenotype was associated with increased risk of P[6] RVGE (Table 3).

Table 3

\begin{tabular}{llll} 
& $\begin{array}{l}\text { Secretor } \mathrm{n}=148 \\
\text { OR }(95 \% \mathrm{CI})\end{array}$ & $\begin{array}{l}\text { Lewis negative } \mathrm{n}=39 \\
\text { OR }(95 \% \mathrm{CI})\end{array}$ \\
\hline P[8] RVGE & $\mathrm{n}=45$ & $\mathrm{n}=4$ \\
$\mathrm{n}=47$ & $7.8(1.8-33.7) \mathrm{p}=\mathbf{0 . 0 0 6}$ & $0.28(0.09-0.83) \mathrm{p}=\mathbf{0 . 0 2 2}$ \\
\hline P[4] RVGE & $\mathrm{n}=36$ & $\mathrm{n}=36$ \\
$\mathrm{n}=38$ & $5.8(1.3-25.2) \mathrm{p}=\mathbf{0 . 0 1 9}$ & $0.17(0.04-0.73) \mathrm{p}=\mathbf{0 . 0 1 7}$ \\
\hline P[6] RVGE & $\mathrm{n}=27$ & $\mathrm{n}=13$ \\
$\mathrm{n}=34$ & $0.99(0.39-2.5) \mathrm{p}=0.98$ & $3.0(1.3-6.7) \mathrm{p}=\mathbf{0 . 0 0 8}$
\end{tabular}

HBGA phenotype and clinical protection

119 RV GE cases (vaccine failures) were compared to 119 agematched community controls. The prevalence of non-secretor phenotype in community controls was $28 \%(20-37 \%)$ and Lewis negative phenotype $26 \%(19-35 \%)$. Non-secretor phenotype was associated with reduced risk of clinical vaccine failure (Table 4).

Table 4

\begin{tabular}{|l|l|l|}
$\begin{array}{l}\text { HBGA } \\
\text { phenotype }\end{array}$ & $\begin{array}{l}\text { Prevalence in } \\
\text { RV GE cases } \\
n, \%(95 \% \mathrm{CI})\end{array}$ & $\begin{array}{l}\text { Odds of rotavirus } \\
\text { vaccine failure } \\
\text { OR }(95 \% \mathrm{Cl})\end{array}$ \\
\hline Non-secretor & $14 / 119,12(7-19 \%)$ & $0.39(0.20-0.75) \mathrm{p}=0.005$ \\
\hline Lewis negative & $24 / 119,20(14-28 \%)$ & $0.70(0.37-1.3) \mathrm{p}=0.265$ \\
\hline
\end{tabular}

\section{Conclusions}

We found no evidence that HBGA phenotype determines vaccine virus shedding or seroconversion. The lower vaccine failure in nonsecretors is due to the relative protection this phenotype confers against the common $P[8]$ and $P[4]$ rotavirus strains. HBGA phenotype distribution is unlikely to contribute significantly to reduced rotavirus vaccine effectiveness in Malawi. 